\title{
A New Method of particle swarm optimization approach for tuning PID controller
}

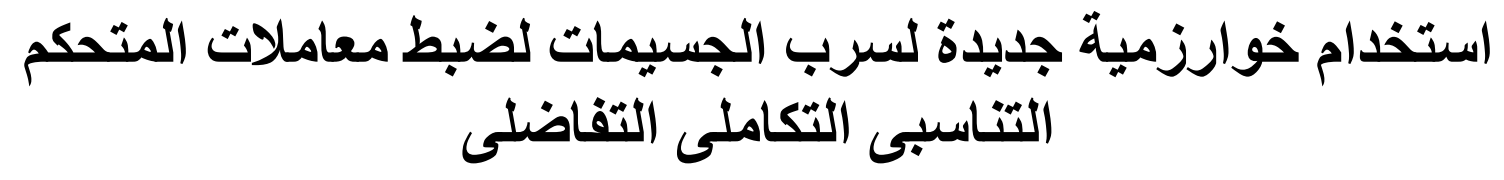

Amal Moharam, Mostafa A. El-Hosseini, Hesham A. Ali

Computers Engineering and Control systems Dept., Faculty of Engineering, Mansura University.

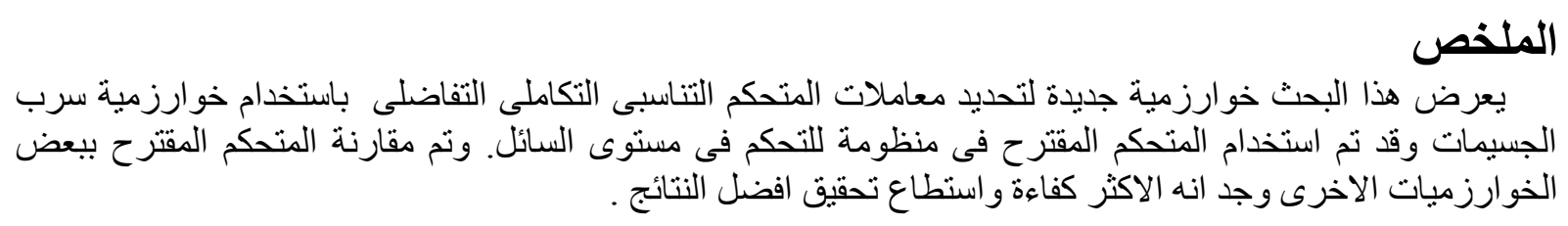

\begin{abstract}
This paper outlines a design method for tuning of PID controller using new algorithm based on Particle Swarm Optimization. The tuned PID controller is used in liquid level system control. Compared to different algorithms; the proposed method was more efficient. It gets the best results.
\end{abstract}

\section{Keywords:}

Particle swarm optimization (PSO); PID controller.

\section{Introduction}

In industrial control system, several control methods such as adaptive control, neural control, and fuzzy control have been used. However, the best known controller is proportional-integral-derivative (PID) controller. PID controller is widely used in the industry because of its simple structure and robust performance.

However, the process of setting the optimal gains of PID controllers is very difficult because many industrial plants have often many problems such time delays, and nonlinearities. Several methods have been proposed for the tuning of PID controllers such as Ziegler and Nichols (Z$N)$ method [1] and Cohen-Coon method.
$\mathrm{Z}-\mathrm{N}$ method is the oldest method and simplest one.

However, it is often hard to determine optimal or near optimal parameters of PID with the Z-N. formula in many industrial plants. Z-N method fails to provide an acceptable performance because it is always provides a large overshoot and settling time, so that the values of the PID parameters are often subsequently refined in accordance with the operator's experience [37].

Recently, many evolutionary computation methods such as genetic algorithm (GA), 
many evolutionary computation methods such as genetic algorithm (GA), differential evolution (DE) and particle swarm optimization (PSO) have been employed to tune PID controller in various plants [2-7].However, the simplicity of PSO (i.e. it is straight forward and has less parameters to be tuned) and its low computational cost with high performance make it commonly used in the industrial applications [43].

PSO is an evolutionary computation technique proposed by Kennedy and Eberhart in [8]. The original intent of PSO is to simulate the social interaction behavior of birds flocking. In PSO, each particle $\mathrm{p}$ in the flock is initialized with randomly chosen velocity $V p$ and position $X p$ in an n-dimensional search space. The PSO concept consists of, at each time step, accelerating each particle towards its own historically best position $\left(\right.$ pbest $\left._{\mathrm{p}}\right)$ and the best position found by the entire swarm (gbest)[9]. The update equations in the PSO are:

$\left.\begin{array}{c}\left.V_{p d}(t+1)=\omega V_{p d}(t)+C_{1} r_{1} \text { pbest }_{p d}-X_{p d}(t)\right)+C_{2} r_{2} \text { (gbest }_{d}- \\ \left.X_{p d}(t)\right) \\ X_{p d}(t+1)=X_{p d}(t)+V_{p d}(t+1)\end{array}\right\}(1)$

Where $r_{1}, r_{2}$ are random numbers uniformly distributed in $[0,1] . C_{1}, C_{2}$ are called acceleration constants, $\omega$ is the inertia weight, and $d(d=1,2, \ldots, n)$ represents the $d$ th dimension of the search space.

Although PSO has fast convergence behavior, there is some deficiency in PSO performance. This is due to that all particles learn from best particle in updating velocities and positions. If best particle located at a local optimum the whole swarm may trap and this would lead to premature convergence.

Various attempts have been made to improve the performance of PSO. One of these attempts is hybridizing PSO with other search techniques [10-17] to benefit from advantages of each algorithm. The hybridization between DE [18] and PSO looks a promising optimizer. This hybridization tries to benefit from good global search capability of DE and high speed convergence of PSO.

$\mathrm{DE}$ is a simple evolutionary algorithm for global optimization proposed by Price and Storn. The DE-variants perturb the current generation population members with the scaled differences of randomly selected and distinct population members. Therefore, no separate probability distribution has to be used for generating the offspring [19]. Easy methods of implementation and negligible parameter tuning made the algorithm quite popular.

In the past decade, numerous hybrids of DE and PSO have been made [20-23]. Particle swarm optimization with differentially perturbed velocity (PSO-DV) is considered one of embedded hybrid algorithms [21]. In this algorithm a differential operator (borrowed from differential evolution) has been tightly coupled with the velocity update scheme of PSO. This differential operator can be considered as additional mutation to guarantee more diversity to PSO and hence avoid local optimum problem. Unlike the PSO scheme, a particle is shifted to a new location only if the new location yields a better fitness value. Due to this restriction, some of the particles may get stagnant in the search space. PSO-DV algorithm tries to solve this problem by replacing these stagnated particles with randomized particles.

In PSO-DV algorithm, DE is embedded in PSO. The operating manners of DE and PSO optimizers cannot be separated explicitly. We cannot separate their contribution to fitness improvement since DE and PSO are integrated into a hybrid optimizer. So, PSO-DV usually implies a higher risk of design failure [20].

Recently, a new PSO approach has been introduced based on aging concept [24][28]. It applies the age index on a particle (or a group of particles) in the swarm. If the particle cannot contribute effectively in searching space (i.e. it gets old), it should be replaced. Thus, the age of the particle is considered as an important indicator of its 
quality. The way of adjusting the life span of a particle and selecting a new one is an important step.

PSO with an aging leader and challengers (ALC-PSO) [28] is an age-based PSO algorithm. ALC-PSO is an attempt to simulate the aging concept in the nature. As the leader of the colony becomes old, it gives the opportunity to another individual to lead the colony. In this algorithm, when the swarm falls in local optima, this means that gbest gets old and cannot lead the swarm. ALC-PSO searches about another promising particle (called a challenger) to replace gbest. To do that, it travels randomly in one way direction for a predetermined number of attempts to select a challenger. The most important feature of ALC-PSO is keeping fast convergence of PSO. However, it is often hard to get acceptable diversity to search more and more promising area of search space.

In this paper, an effective approach called hybrid Differential Evolution and Particle Swarm Optimization with an Aging Leader and Challengers (ALC-PSODE) is presented. ALC-PSODE modifies PSODV by using another mutation borrowed from ALC-PSO (ashelping agent) to solve stagnation of particles problem and improve the performance of PSO-DV. Using one dimensional mutation of ALCPSO can prevent gbest moving to the position of randomly mutated particle. This can protect the swarm from unacceptable divergence. To validate ALC-PSODE algorithm, it first tested on five benchmark functions. Then it is also used for tuning PID controller.

The rest of the paper is organized as follows. Section 2 reviews related work. Section 3 develops the ALC-PSODE algorithm in detail. Section 4 experimentally validates the ALC-PSODE and compares it with five evolutionary algorithms on five benchmark functions. Section 5 describes the linearized model of liquid level system. Section 6 describes the application of ALC-PSODE to tune the PID controller and test it against various controllers. Finally, section 7 concludes this paper.

\section{Related work}

To solve the premature convergence problem of PSO, many algorithms that are based on adding a new mutation to PSO have been introduced as PSO-DV [21] and ALC-PSO [28]. In the PSO-DV algorithm [21], for each particle $p$ in the swarm two other distinct particles, say $\mathrm{p} 1$ and $\mathrm{p} 2(\mathrm{p} \neq$ $\mathrm{p} 1 \neq \mathrm{p} 2$ ), are selected randomly. The difference between their positional coordinates is taken as a difference vector $\delta$ :

$\delta=X_{p 2}-X_{p 1}$

Then the d-th velocity component $(1<\mathrm{d}<$ number of dimensions) of the target particle $\mathrm{p}$ is updated as:

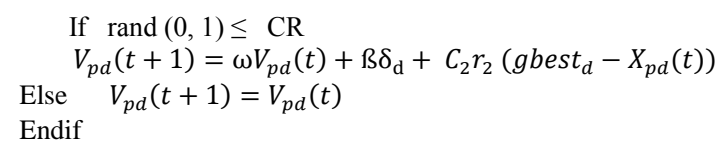

Where CR is the crossover probability, $\beta$ is a scale factor in $[0,1]$ and $\delta_{d}$ is the d-th $\delta$ component .The aim of using vector differential operator is to produce some additional exploration capability. The next step is creating a new trial location $\operatorname{Tr}_{p}$ for the particle:

$$
\operatorname{Tr}_{p}=X_{p}(t)+V_{p}(t+1)
$$

Then the algorithm selects between the trial vector $\operatorname{Tr}_{p}$ and position vector $X_{p}(t)$. It selects the vector which has better fitness value. Due to this selection strategy, some particles may not be able to find better position (i.e. they get stagnant). The algorithm tackles this stagnation by shifting these particles to a new locationusing a random mutation. However, this means slow and unstable convergence especially if there are a large number of stagnated particles.

Some PSO-DV variants have been proposed to improve its performance. The simplest one is APSO-DV algorithm (particle swarm optimization with differentially perturbed velocity hybrid algorithm with adaptive acceleration 
coefficient) [29].The main objective of APSODV is to use adaptive acceleration coefficient for updating the positions of the particles of PSODV to accelerate the search for the global solution. In general, tuning the parameters can improve the performance of PSO-DV, but this is not a general case.

Another modified PSO-DV algorithm is evolving ant direction particle swarm optimization with differentially perturbed velocity (EADPSODV) [30]. In this approach, ant colony search is utilized by the EADPSODV algorithm to find a suitable mutation operator for PSODV. Genetic algorithm method is employed to evolve the ant colony parameters.

ALC-PSO [28] is a new technique to tackle the shortcoming of PSO. Its key idea is based on invoking a new mutation notion. When gbest fails to lead the swarm, all swarm particles learn from another particle (i.e. a challenger). Hence, the diversity can be restored again after it had been lost. The steps of ALC-PSO algorithm can be summarized as follows:

1. First, initialize randomly the position and velocity of all particles ( $p=$ $[1,2, \ldots . M])$ in the swarm, set gbest as a Leader, initialize the age of the Leader $\theta=0$, set the lifespan of the Leader to an initial value (e.g.lifespan=30).

2. In the beginning of the run; in each generation, updating the velocity, position, pbest and Leader as in the PSO algorithm.

3. Adjust life span as will be discussed later and increase the age of the Leader $\theta$ by 1 .

4. Check if the Leader become aged (i.e. age of the Leader> lifespan), then go to step 5. Else, go to step 7.

5. Generate a challenger which is different from the previous Leader in only one dimension (i.e. to keep some good characteristics of it).

6. Test the challenger, if it can improve at least one pbest; then replace the Leader by the challenger. Set the age of the Leader $\theta=0$; set the lifespan of the
Leader to an initial value. If the challenger fails in testing, then the old leader remains leading the swarm.

7. Check if stop condition is satisfied, then terminate the algorithm .Else, go to step 2.

The main component of ALC-PSO that determines its performance is life span. The lifespan is adjusted according to the leader's ability to improve the swarm. To determine leader's ability, ALC-PSO observes 3 indexes during a Leader's lifetime (the decision tree for the lifespan controller is shown in Fig. 1) ${ }^{1}$ :

1- Improvement of gbest.

If $f(\operatorname{gbest}(\theta))-f(\operatorname{gbest}(\theta-1))<0$; then the current Leader have a good leading power and it is expected to have the ability to improve the swarm in the following generations. So the lifespan of the Leader is elongated (i.e. lifespan is increased by 2).

2- Improvement of collective pbest . $^{\text {. }}$

If $\sum_{p=1}^{M} f\left(\right.$ pbest $\left._{p}(\theta)\right)-\sum_{p=1}^{M} f\left(\right.$ pbest $\left._{p}(\theta-1)\right)<0$; this means that the historically gbest is not improved but the Leader is still able to improve at least one pbest $_{p}$ position. This situation implies that the current Leader still has the ability to improve the swarm (but at less degree) in the following generations. So the lifespan of the Leader is increased by 1 .

3- Improvement of Leader.

If $\quad($ Leader $(\theta))-f($ Leader $(\theta-1))<0$; this means that the current Leader fails to lead any of the particles in the swarm toward better positions. However, the Leader still has the ability to improve itself. Hence, the ability of the Leader to improve the swarm in the following generations is dubious. Therefore, thelifespan of the Leader remains unchanged.

If there is no improving in the above three indexes, then the current Leader is expected to have no ability to improve the swarm in the following generations. So the lifespan of the Leader is shortened (i.e. lifespan is reduced by 1).Indeed, this decreasing in lifespan means that the leader 
is getting old by higher rate (i.e. the moving towards replacing the Leader).

\section{Proposed ALC- psodealgorithm}

In the beginning of the run, ALCPSODE is as PSO-DV in updating the velocity and position. If the swarm traps, a simple mutation is borrowed from ALCPSO to tackle PSO-DV deficiency. This mutation is done by making the swarm learning from another particle which differs from the old leader in one dimension. This one dimension mutation can introduce the diversity again to PSODV and moreover keeping acceptable convergence. The updated velocity equation is changed to:

$V_{p d}(t+1)=\omega V_{p d}(t)+\beta \delta_{\mathrm{d}}+C_{2} r_{2}\left(\right.$ Leader $\left._{d}-X_{p d}(t)\right)$

The steps of ALC-PSODE algorithm for minimizing the function $f()$ is shown in Fig. (2).

\section{Test functions and experimental results}

Real problems nowadays are more and more complex. Their objective functions are often multimodal with peaks, valleys, channels, and flat hyper planes of different heights. Solving these types of problems, which are classified as global optimization problems, to optimality undoubtedly, becomes a true challenge. Test functions have many characteristics for simulating the complexity of most real applications. For example; multimodal functions are used to test the ability of an algorithm to escape from any local minimum. If the exploration process of an algorithm is poorly designed, then it cannot search the function landscape effectively. This, in turn, leads to an algorithm getting stuck at a local minimum.

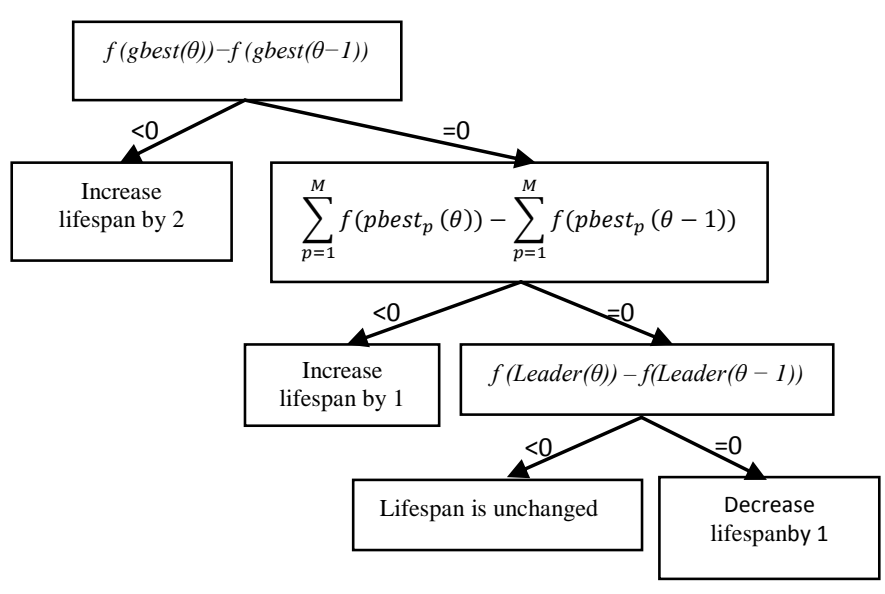

Fig.1. Decision tree for the lifespan controller to adjust the lifespan (The Age of the leader $\theta=$ $1,2, \ldots$, , lifespan) 


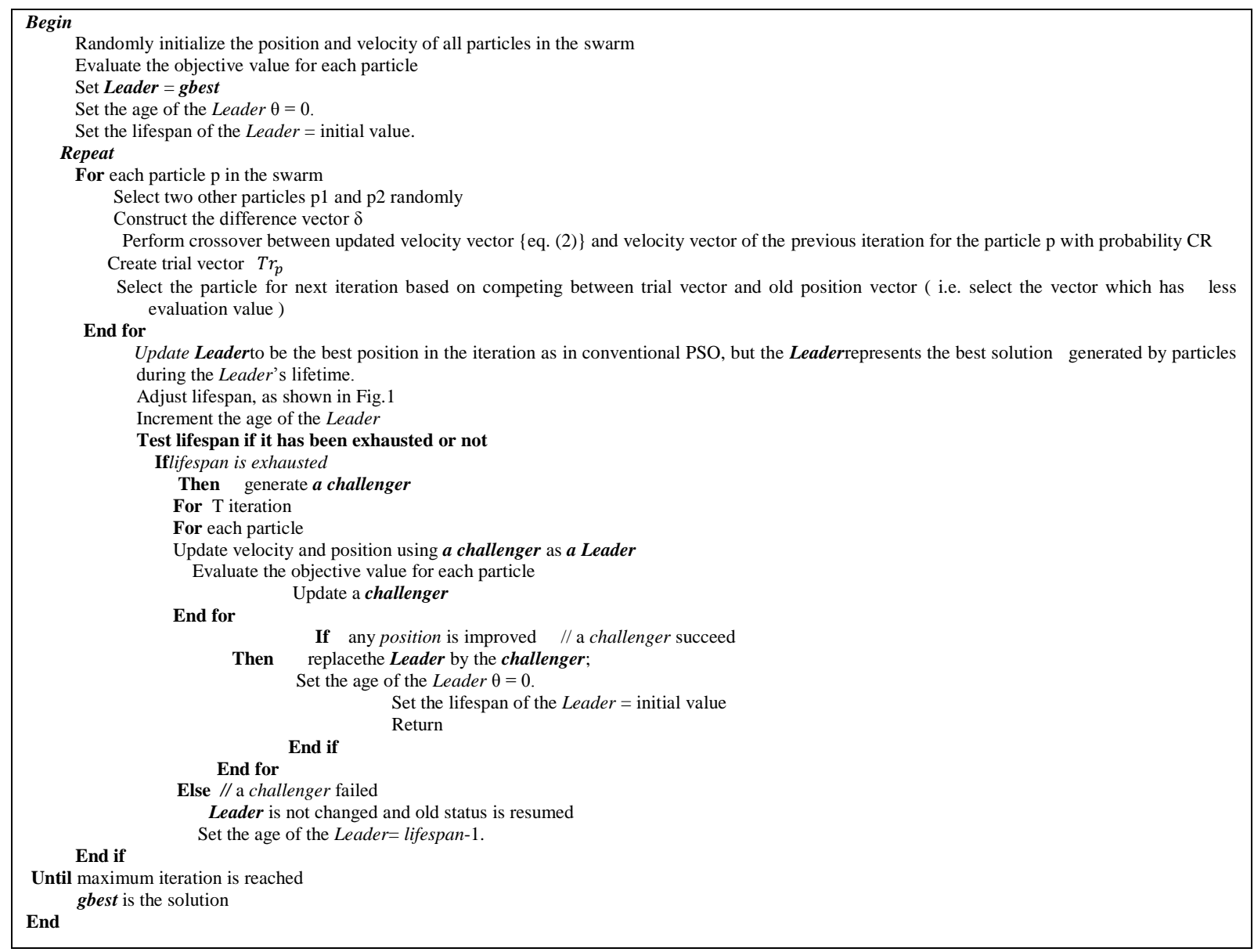

Fig. 2. Pseudo code of ALC-PSODE algorithm

\subsection{Benchmark and experimental settings}

ALC-PSODE is tested against five wellknown benchmarks functions to evaluate the performance of the proposed algorithm. These test functions are shown in Table 1. The first two test functions are unimodal, having only one minimum. The others are multimodal with many local optima.

The proposed algorithm has been tested against the canonical PSO,GA, DE (rand/1/bin), ALC-PSO and PSO-DV, algorithms. The number of dimensions of all the test functions is set to $n=30$.The population size $=100$.

In the experiment, the parameters of PSO, ALC-PSO and ALC-PSODE are set as follows: the acceleration coefficients $\mathrm{c}_{1}=\mathrm{c}_{2}$ $=2.0$, the inertia weight $\omega=0.4$ [28], the initial value of lifespan $=30$, the number of steps $\mathrm{T}$ for evaluating the leading power of a challenger is set to $\mathrm{T}=3$ and the legal velocity range is set to $50 \%$ of the search range. In the case of PSO-DV and ALCPSODE, we choose the crossover constant $\mathrm{CR}=0.9$ and the scale factor $B$ is linearly varying from 0.9 at the beginning of the search to 0.4 at the end of the search. For $\mathrm{DE}$, the crossover constant $\mathrm{CR}=0.9$ and the scale factor $F=0.8$. In the case of $G A$, roulette wheel selection operator, single point crossover and adaptive mutation were employed [43,44].

To reduce statistical errors, each test is repeated 50 times independently. During each run, a maximum number of 10000 function evaluations (FEs) are used. The reliability of search is reflected by the "success\%" in Table 2, which stands for the percentage of the successful runs that acceptable solutions are found. Each run is considered to be success if and only if the best solution found by an algorithm achieves the predetermined accuracy level in Table 1. 
Table $1^{*}$

Test Functions

\begin{tabular}{|c|c|c|c|}
\hline \multicolumn{2}{|c|}{ Test Function } & $\begin{array}{l}\text { Range of } \\
\text { search }\end{array}$ & $\begin{array}{l}\text { Optimum } \\
\text { 土accuracy }\end{array}$ \\
\hline \multicolumn{4}{|c|}{ Unimodal Functions (2) } \\
\hline Sphere & $f_{1}(x)=\sum_{i=1}^{n} x_{i}^{2}$ & {$[-100,100]$} & $0 \pm 0.1$ \\
\hline $\begin{array}{l}\text { Rosenbroc } \\
\text { k's }\end{array}$ & $f_{2}(x)=\sum_{i=1}^{n-1}\left[100\left(x_{i+1}-x_{i}^{2}\right)^{2}+\left(x_{i}-1\right)^{2}\right]$ & {$[-10,10]$} & $0 \pm 100$ \\
\hline \multicolumn{4}{|c|}{ Multimodal Functions (3) } \\
\hline Rastrigin & $f_{3}(x)=\sum_{i=1}^{n}\left[x_{i}^{2}-10 \cos \left(2 \pi x_{i}\right)+10\right]$ & {$[-5.12,5.12]$} & $0 \pm 100$ \\
\hline Ackley & 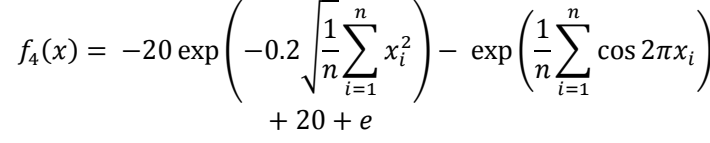 & {$[-32,32]$} & $0 \pm 0.1$ \\
\hline Griewank & $f_{5}(x)=\frac{1}{4000} \sum_{i=1}^{n} x_{i}^{2}-\prod_{i=1}^{n} \cos \left(\frac{x}{\sqrt{i}}\right)+1$ & {$[-600,600]$} & $0 \pm 0.1$ \\
\hline
\end{tabular}

*For more details about test functions browse: http://www3.ntu.edu.sg/home/EPNSugan/

\subsection{Comparison results}

The following performance measures are used for our comparative study:

- Success rate (number of successful runs/total runs).

- Quality of the final solution.

- Speed of convergence towards the optimal solution.

In Table 2, the best solution, worst solution, mean and standard deviation yielded by different algorithms for 50 independent runs are reported. It can be seen that only ALCPSODE, ALC-PSO and GA are able to find acceptable solutions with a $100 \%$ successful rate. We compare between ALC-PSODE and other two algorithms using two-sample $t$ tests. Two-sample t-test is a hypothesis testing method used for assessing whether the means of two independent samples are statistically different from each other [38, 39]. According to the results of $t$-tests (The difference between two samples is significant at level $\alpha=0.05$, sample size $=50$ and degrees of freedom $=98$ ), ALC-PSODE significantly outperforms ALC-PSO on $f_{2}, f_{5}$. Also, ALC-PSODE significantly outperforms GA on the first four functions.
In Fig. 3 we have graphically presented the rate of convergence (for an average run) of all methods for all functions. Although, the performance of all algorithms were comparable to each other regarding to all test bench mark functions, the performance of ALC-PSODE is super exceeded the performance of other algorithms regarding to $f_{2}, f_{3}$ and this is shown in Fig. 3. These results show that the proposed algorithm leads to significant improvement in most cases.

\section{A case study: liquid level system control}

After validation of ALC-PSODE algorithm on test benchmark functions, it will be used for tuning PID controller. This controller, for simplicity, is called ALCPSODE-PID controller. For testing on real application, ALCPSODE-PID controller is used for controlling 3 tanks liquid level system which is a typical nonlinear complexcontrol system. Liquid level control is very important in many industrial applications as in water purification systems, 
industrial chemical processing and boilers in all the industries.

\subsection{Linearized model of three tanks liquid level system}

A simple structure of the water tank is shown in Fig.4. In this device, there are three tanks: $\operatorname{tank} \mathrm{A}, \operatorname{tank} \mathrm{B}$, and tank $\mathrm{C}$. Tank D is the main tank, which provides the water for the pump. The control actuator is an electric valve. The control signal will change the open range of the electric valve from $0 \%$ to $100 \%$. Different open range of electric valve means different water flow rate.

Table2*

Results obtained for 50 runs

\begin{tabular}{|c|c|c|c|c|c|c|c|}
\hline function & & PSO & GA & $\mathrm{DE}$ & PSO-DV & ALC-PSO & ALC-PSODE \\
\hline \multirow{6}{*}{$f_{1}$} & best & $3.2878 \mathrm{e}-282$ & $1.8600 \mathrm{e}-009$ & $1.0432 \mathrm{e}-004$ & $8.6345 \mathrm{e}-037$ & $4.4586 \mathrm{e}-285$ & $8.8404 \mathrm{e}-056$ \\
\hline & worst & $3.3699 \mathrm{e}-269$ & $1.3500 \mathrm{e}-008$ & 0.0024 & $4.7471 \mathrm{e}-032$ & $2.7508 \mathrm{e}-273$ & $1.5182 \mathrm{e}-033$ \\
\hline & mean & $1.0967 \mathrm{e}-270$ & $5.7207 \mathrm{e}-009$ & $8.1551 \mathrm{e}-004$ & $2.7634 \mathrm{e}-033$ & $1.6095 \mathrm{e}-274$ & $3.5322 \mathrm{e}-035$ \\
\hline & Std deviation & 0 & $3.1072 \mathrm{e}-009$ & $5.1190 \mathrm{e}-004$ & $8.3100 \mathrm{e}-033$ & 0 & $2.1480 \mathrm{e}-034$ \\
\hline & Success $\%$ & 100 & 100 & 100 & 100 & 100 & 100 \\
\hline & t test & & -13.0186 & & & 1.1628 & \\
\hline \multirow{6}{*}{$f_{2}$} & best & 0.1255 & $4.6300 \mathrm{e}-005$ & 16.9436 & $2.3484 \mathrm{e}-022$ & $1.6790 \mathrm{e}-004$ & $2.4479 \mathrm{e}-021$ \\
\hline & worst & 96.9987 & 6.4701 & 22.0152 & $8.3210 \mathrm{e}-004$ & 77.7372 & $1.0906 \mathrm{e}-005$ \\
\hline & mean & 33.4918 & 1.3229 & 19.6060 & $1.6706 \mathrm{e}-005$ & 17.3852 & $3.6305 e-007$ \\
\hline & Std deviation & 30.3198 & 2.2989 & 1.1931 & $1.1767 \mathrm{e}-004$ & 15.9752 & $1.6084 \mathrm{e}-006$ \\
\hline & Success \% & 92 & 100 & 100 & 100 & 100 & 100 \\
\hline & t test & & -4.0690 & & & -7.6952 & \\
\hline \multirow{6}{*}{$f_{3}$} & best & 16.9143 & 2.9849 & 57.0098 & $\mathbf{0}$ & 0 & $1.7764 \mathrm{e}-015$ \\
\hline & worst & 97.5765 & 10.9446 & 99.5367 & 99.4949 & $6.8350 \mathrm{e}-008$ & $1.2736 \mathrm{e}-012$ \\
\hline & mean & 64.7968 & 6.4009 & 82.4987 & 50.7627 & $1.3677 \mathrm{e}-009$ & $4.7855 \mathrm{e}-014$ \\
\hline & Std deviation & 20.2555 & 2.3324 & 12.3051 & 25.8691 & $9.6661 \mathrm{e}-009$ & $1.7978 \mathrm{e}-013$ \\
\hline & Success \% & 90 & 100 & 34 & 98 & 100 & 100 \\
\hline & $t$ test & & -19.4054 & & & -1.0005 & \\
\hline \multirow{6}{*}{$f_{4}$} & best & $4.4409 \mathrm{e}-015$ & $2.9400 \mathrm{e}-005$ & $5.2288 \mathrm{e}-010$ & $1.1546 \mathrm{e}-014$ & $4.4409 \mathrm{e}-015$ & $7.9936 \mathrm{e}-015$ \\
\hline & worst & $7.9936 \mathrm{e}-015$ & $6.7400 \mathrm{e}-005$ & $3.4389 \mathrm{e}-008$ & $2.2204 \mathrm{e}-014$ & $7.9936 \mathrm{e}-015$ & $1.5099 \mathrm{e}-014$ \\
\hline & mean & $7.1794 \mathrm{e}-015$ & $5.3303 \mathrm{e}-005$ & $7.5172 \mathrm{e}-009$ & $1.7838 \mathrm{e}-014$ & $7.6383 e-015$ & $9.5568 \mathrm{e}-015$ \\
\hline & Std deviation & $1.5090 \mathrm{e}-015$ & $9.3242 \mathrm{e}-006$ & $1.1973 \mathrm{e}-008$ & $3.6819 \mathrm{e}-015$ & $1.0766 \mathrm{e}-015$ & $2.9733 \mathrm{e}-015$ \\
\hline & Success\% & 96 & 100 & 14 & 96 & 100 & 100 \\
\hline & $t$ test & & -40.4227 & & & 4.2 & \\
\hline \multirow{6}{*}{$f_{5}$} & best & $\mathbf{0}$ & $4.4400 \mathrm{e}-011$ & $3.2851 \mathrm{e}-004$ & 0 & 0 & $\mathbf{0}$ \\
\hline & worst & 0.0638 & $4.5900 \mathrm{e}-010$ & 0.0213 & 0.0638 & 0.0515 & 0.0344 \\
\hline & average & 0.0147 & $2.3658 \mathrm{e}-010$ & 0.0037 & 0.0123 & 0.0100 & 0.0031 \\
\hline & Std deviation & 0.0159 & $9.5324 \mathrm{e}-011$ & 0.0044 & 0.0135 & 0.0110 & 0.0060 \\
\hline & Success \% & 100 & 100 & 100 & 100 & 100 & 100 \\
\hline & $\mathrm{t}$ test & & 3.6534 & & & -3.8939 & \\
\hline \multicolumn{3}{|c|}{ ALC-PSODE obtains significantly better results } & 4 & & & 2 & \\
\hline \multicolumn{3}{|c|}{ ALC-PSODE obtains significantly worse results } & 1 & & & 1 & \\
\hline
\end{tabular}

* Best solution, worst solution, mean and standard deviation are calculated for only success runs.

* Bold numbers indicate the featured results (i.e. success rate $=\% 100$, significantly better results for $t$ test of ALC-PSODE and the minimum (best) results in best, worst, mean and standard deviation ).

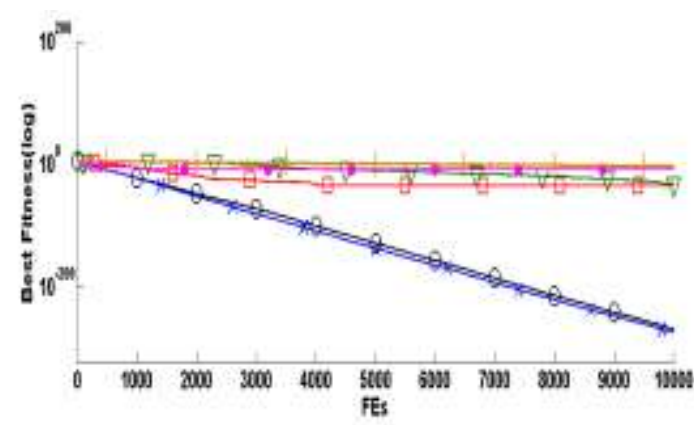

(a)
From the simple structure of Fig.4, the main principle of water level system can be explained as following: Driven by pump, the water in tank D is piped to tank A, B and C. Water level will be measured by pressure sensor at bottom of each tank. The difference between actual level and set-point value is calculated. Then the control input can be obtained by PID algorithm. The control input will be feedback to electric valve to change the flow rate, and then the level of water in each tank can be controlled [31]. 


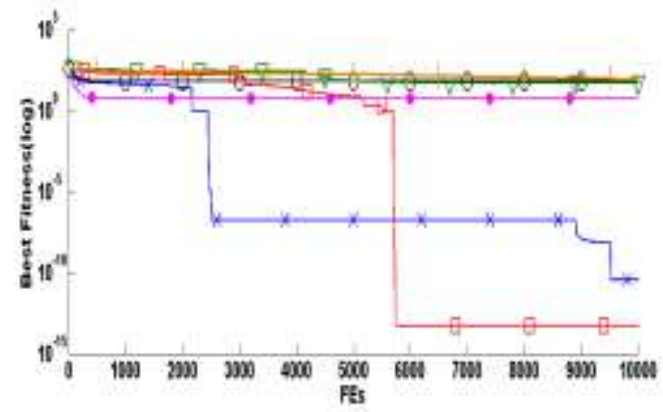

(c)

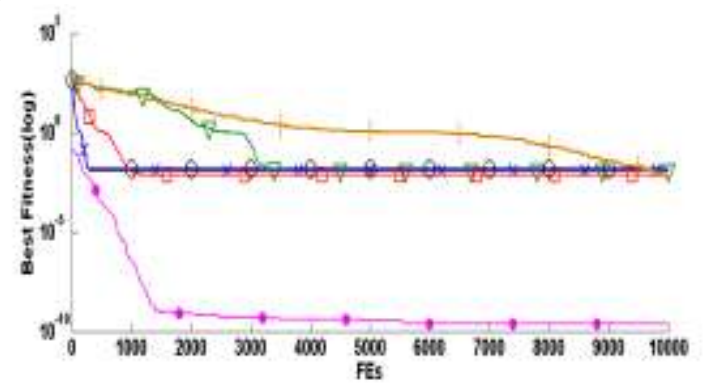

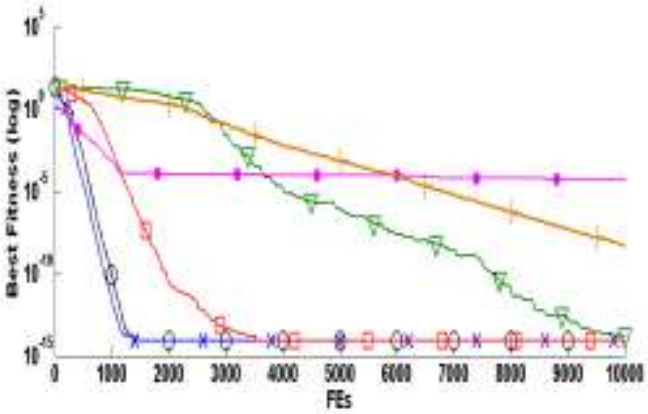

(d)

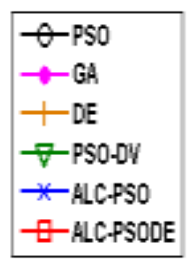

(e)

Fig. 3. Convergence graphs on test functions (a) $f_{1}$. (b) $f_{2}$. (c) $f_{3}$. (d) $f_{4}$. (e) $f_{5}$

To analyze the mechanism of Water Level System, the following symbols should be considered first:

$Q_{1}$-inflow of water tank;

$Q_{2}$ - outflow of water tank;

A- area of tank's section;

$\mathrm{VO}$ - valve's opening range;

LH - water level height;

$K_{1}$-valve flux proportional coefficient;

$K_{2}$-flux proportional coefficient

Here only the dynamic characteristic of Tank $\mathrm{C}$ is analyzed. Consider that $\mathrm{A}, K_{1}, K_{2}$ are time invariant parameters, $V O_{0}, L H_{0}$ are the input and output value of an equilibrium point, then from Fig.5 we have:

$$
\begin{gathered}
Q_{2}-Q_{1}=A(d L H / d t) \\
Q_{1}=K_{1} \times V O \\
Q_{2}=K_{2} \times \sqrt{L H}
\end{gathered}
$$

From eq.(3),eq.(4) and eq.(5), it can be seen that the water level is a system with nonlinearity. Around the equilibrium point $\left(V O_{0}, L H_{0}\right)$, eq.(5) can be rewritten approximately as:

$$
\frac{Q_{2}}{L H} \approx \frac{K_{2}}{\sqrt{L H_{0}}}=K_{3}
$$

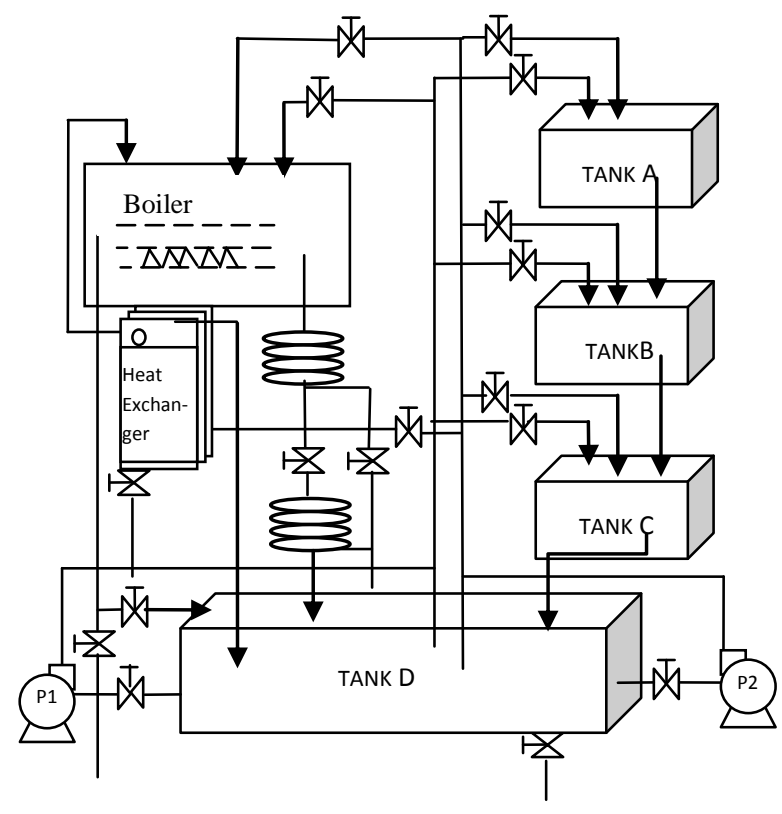

Fig.4. Simple structure of the water level system

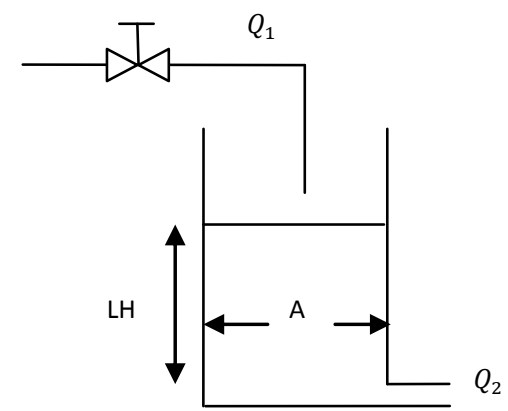

Fig.5. Water level system with control valve 
Calculate the Laplace Transformation of eq. (3), eq.(4) and eq.(6) .From the Laplace equations, linear model (around the equilibrium point $\left.\left(\mathrm{VO}_{0}, \mathrm{LH}_{0}\right)\right)$ with input $V O$, output $L H$ can be given as below:

$$
\mathrm{G}(\mathrm{s})=\frac{\mathrm{LH}(\mathrm{s})}{\mathrm{VO}(\mathrm{s})}=\mathrm{Ke}^{-\tau \mathrm{s}} /(\mathrm{Ts}+1)
$$

where $K=K_{1} / K_{3}, \quad T=A / K_{3}, \tau$ is the inherent time delay.

Consider that $\mathrm{A}=20, K_{1}=2, K_{2}=5, \tau=0$; the system will come out to be third order dynamics model. Desired transfer function of three tank water level system is [32]:

$$
\frac{H(s)}{Q(s)}=\frac{1}{64 s^{3}+9.6 s^{2}+0.48 s+0.008}
$$

\subsection{Liquid level system with PID controller}

ALC-PSODE algorithm is used to find for the optimal PID parameters $\left[\mathrm{K}_{\mathrm{p}}, \mathrm{K}_{\mathrm{i}}\right.$, $\mathrm{K}_{\mathrm{d}}$ ] that will minimize the objective function ISE (integral of squared-error). The ISE performance criterion formula is as follows:

$$
I S E=\int_{0}^{\infty}(r(t)-h(t))^{2}=\int_{0}^{\infty} e^{2}(t) d t
$$

Three Tanks Liquid Level System compensated with a PID controller block diagram is shown in Fig. 6.

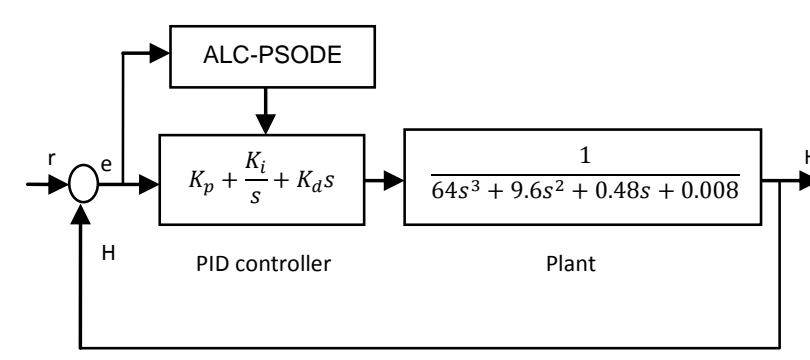

Fig.6. Block diagram of Intelligent PID controller

\section{Results}

\subsection{Performance of ALC-PSODE controller}

The following PSO parameters are used for verifying the performance of the various PSO-PID controllers:

Inertia weight $\omega=0.4$ [28];

Acceleration constant $\mathrm{c} 1$ and $\mathrm{c} 2=2$;

Crossover Constant $\mathrm{CR}=0.8$;
Scale Factor $\beta=0.8$ [28]

For GA, roulette wheel selection operator, single point crossover and adaptive mutation were employed. In the case of DE, the crossover constant $\mathrm{CR}=0.9$ and the scale factor $\mathrm{F}=0.8$ [28].

We performed 30 independent runs for various controllers with different random numbers, different swarm size and different number of generations to observe the variation in their evaluation values. Each run is considered to be success if and only if the best solution (i.e. of the objective function ISE) found by an algorithm is less than $30^{2}$. In addition, the best, worst, and average evaluation values were obtained. Results were shown in Table 3. As can be seen, PSO variants can get success rate equal $100 \%$ in all cases. GA and DE fail in some runs to get successful evaluation values. This result is due to the internal structure of GA and DE that degrades their performance [33, 34, 41]. In GA; with continue of generations; crossover usually has less effect, and the resulting movements are relatively smaller. This cause a premature convergence and loss of diversity [34]. Also, DE is easy to drop into local optima because of its fast convergence. To solve premature convergence problem of $\mathrm{DE}$, it is desired to increase the population size for increasing the diversity but this will increase the computation time. So, hybrid DE is always used to overcome its drawback [41].

rom the table, all algorithms can get the best solution except PSO. We can observe that ALC-PSO can get betterresult than PSO but it cannot solve the premature convergence problem of PSO completely. This was exactly done when testing benchmark function. The evaluation values of the ALCPSODE-PID controller generated fluctuation in a very small range (std. dev. = 8.2827e-015), thus verifying that the ALCPSODE-PID controller has better convergence characteristic and hence it is the most robust algorithm. The simulation results that showed the best solution (i.e. the comparison has been done using transientresponse specifications [40] of the system in 
addition to ISE) and step response of the three tanks liquid level system for best resultswere summarized in Table 4 and Fig.7 respectively.

\subsection{Convergence characteristic}

As can be seen from Table 3, only PSO variants can get $\% 100$ success rate. So, we will study the convergence characteristics of PSO variants for least population size. For fair comparisons, the same seed of random numbers in initialization of the population has been used. Convergence characteristic of the various PSO-PID controllers is shown in Fig.8. We can see that although conventional PSO has fast convergence characteristics as expected, it cannot get the best result. Fig. 8 shows that PSO-DV, ALC-PSO and ALCPSODE can get the best result but ALCPSODE has the best convergence among them. Through about 15 iterations (15 generations), the ALCPSO-DEmethod can achieve fast convergence and obtain good evaluation value.

To study convergence behavior of ALCPSODE clearly, we observe the variation in its best evaluation values against various successful algorithms for 30 runs. The results are shown in Fig. 9. The results show that ALC-PSODE generated fluctuation in a small range, thus verifying that ALCPSODE has better convergence characteristic. These discussed results show that the ALCPSODE-PID controller can search optimal PID controller parameters efficiently and quickly.

Table 3

Results obtained for 30 run

\begin{tabular}{|c|c|c|c|c|c|c|c|}
\hline $\begin{array}{c}\text { Different } \\
\text { PID controller }\end{array}$ & 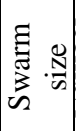 & 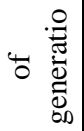 & best & worst & average & $\begin{array}{l}\text { Standard } \\
\text { deviation }\end{array}$ & $\begin{array}{c}\text { Success } \\
\%\end{array}$ \\
\hline PSO & \multirow{6}{*}{20} & \multirow{12}{*}{50} & 8.7016 & 28.020 & 9.3455 & 3.5271 & 100 \\
\hline GA & & & 7.7925 & 28.0392 & 8.9846 & 3.9128 & 90 \\
\hline$\overline{\mathrm{DE}}$ & & & 7.7922 & 10.5203 & 10.1440 & 0.9574 & 96.67 \\
\hline PSO-DV & & & 7.7922 & 7.7922 & 7.7922 & $1.49 \mathrm{e}-07$ & 100 \\
\hline ALC-PSO & & & 7.7922 & 8.7016 & 8.1605 & 0.4497 & 100 \\
\hline ALC-PSODE & & & 7.7922 & 7.7922 & 7.7922 & $1.8 \mathrm{e}-010$ & 100 \\
\hline $\mathrm{PSO}$ & \multirow{6}{*}{30} & & 8.7016 & 8.7016 & 8.7016 & $8.47 \mathrm{e}-014$ & 100 \\
\hline GA & & & 7.7922 & 10.8067 & 8.0341 & 0.6064 & 100 \\
\hline $\mathrm{DE}$ & & & 7.7922 & 10.5203 & 9.5200 & 1.3372 & 100 \\
\hline PSO-DV & & & 7.7922 & 7.7922 & 7.7922 & $3.40 \mathrm{e}-09$ & 100 \\
\hline ALC-PSO & & & 7.7922 & 8.7016 & 7.8638 & 0.2301 & 100 \\
\hline ALC-PSODE & & & 7.7922 & 7.7922 & 7.7922 & $2.01 \mathrm{e}-011$ & 100 \\
\hline PSO & \multirow{6}{*}{20} & \multirow{12}{*}{100} & 8.7016 & 24.423 & 9.2256 & 2.8704 & 100 \\
\hline GA & & & 7.7922 & 8.3474 & 7.8358 & 0.1230 & 96.67 \\
\hline $\mathrm{DE}$ & & & 7.7922 & 10.5203 & 9.9747 & 1.1099 & 100 \\
\hline PSO-DV & & & 7.7922 & 7.7922 & 7.7922 & $2.85 \mathrm{e}-07$ & 100 \\
\hline ALC-PSO & & & 7.7922 & 8.7016 & 7.9553 & 0.3020 & 100 \\
\hline ALC-PSODE & & & 7.7922 & 7.7922 & 7.7922 & $5.62 \mathrm{e}-015$ & 100 \\
\hline PSO & \multirow{6}{*}{30} & & 8.7016 & 8.7016 & 8.7016 & $4.26 \mathrm{e}-014$ & 100 \\
\hline GA & & & 7.7922 & 7.8157 & 7.7932 & 0.0043 & 100 \\
\hline $\mathrm{DE}$ & & & 7.7922 & 10.5203 & 9.8838 & 1.1736 & 100 \\
\hline PSO-DV & & & 7.7922 & 7.7922 & 7.7922 & $2.07 \mathrm{e}-08$ & 100 \\
\hline ALC-PSO & & & 7.7922 & 8.6602 & 7.8788 & 0.2629 & 100 \\
\hline ALC-PSODE & & & 7.922 & 7.922 & 7.922 & $8.28 \mathrm{e}-015$ & 100 \\
\hline
\end{tabular}


Table 4

Best solution using various controllers

\begin{tabular}{|c|c|c|c|}
\hline & $\begin{array}{c}\text { Ziegler and } \\
\text { Nichols }\end{array}$ & PSO & $\begin{array}{c}\text { GA, DE, } \\
\text { PSO-DV, ALC- } \\
\text { ALC-PSODE }\end{array}$ \\
\hline $\mathrm{K}_{\mathrm{p}}$ & 0.03843444 & 0.0528 & $\mathbf{0 . 0 4 1 9}$ \\
\hline $\mathrm{K}_{\mathrm{i}}$ & .0010588 & 0.0003 & $\mathbf{0 . 0 0 0 9}$ \\
\hline $\mathrm{K}_{\mathrm{d}}$ & 0.17396084 & 1 & $\mathbf{1}$ \\
\hline ISE & 32.7064 & 8.7016 & $\mathbf{7 . 7 9 2 2}$ \\
\hline Overshoot & 58.5408 & 15.4004 & $\mathbf{1 2 . 4 6 7 1}$ \\
\hline Rise Time & 18.1142 & $\mathbf{1 2 . 0 5 7 2}$ & 12.7622 \\
\hline Settling Time & 476.6251 & 267.5225 & $\mathbf{6 4 . 2 1 6 4}$ \\
\hline Peak & 1.6262 & 1.1457 & $\mathbf{1 . 1 2 4 7}$ \\
\hline Peak Time & 48.5000 & $\mathbf{2 5}$ & 26.6000 \\
\hline
\end{tabular}

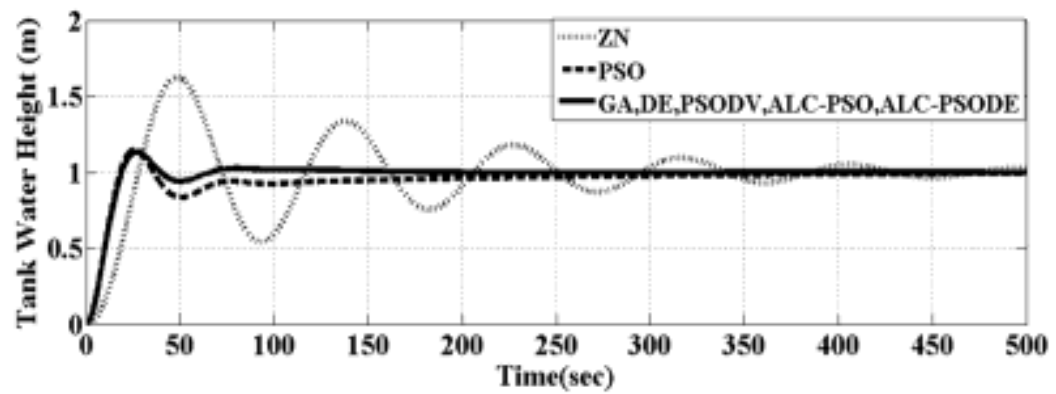

Fig.7 Step response of 3 tanks liquid level system with the various PID controllers for best result.

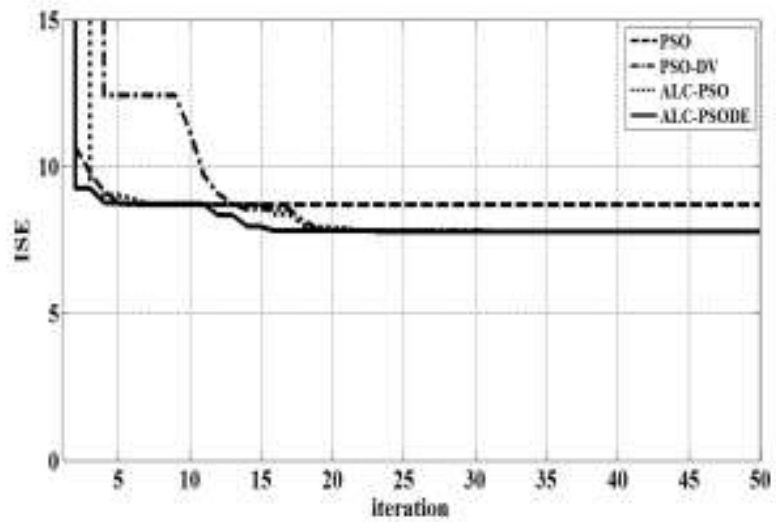

Fig. 8 Convergence tendency of best particle for various PSO-PID controllers (popultion size $=20$, number of generatons $=50$ )

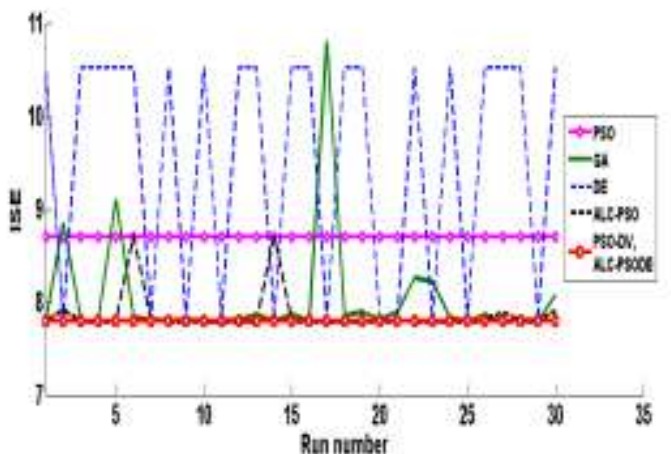

(a) popultion size $=30$, number of generatons $=50$

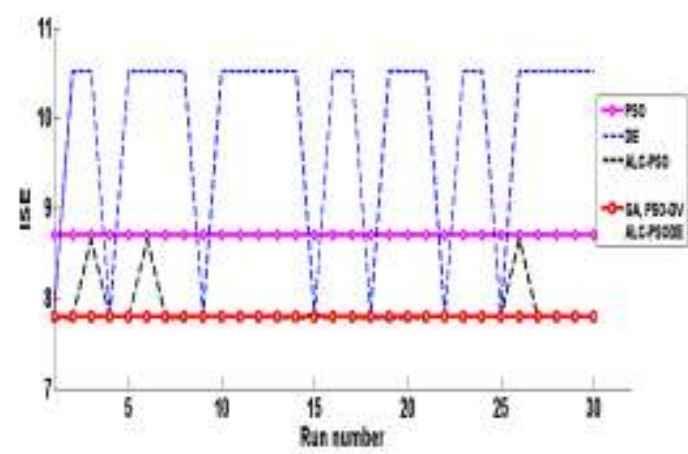

(b) popultion size $=30$, number of generatons $=100$

Fig.9 Comparison of best values of various PID controllers for 30 successful runs 


\section{Conclusion and future works}

In this paper a new PSO variant called ALCPSODE has been presented and has been shown to improve performance in a statistically meaningful way. The new method has been compared against different PSO variants, GA, DE using five well known benchmarks functions. Then the new algorithm has been used in tuning PID controller to control three tanks liquid level system which is typical nonlinear problem. The ALC-PSODE- PID controller is compared against various PID controllers; the results show that the proposed controller can perform an efficient search for the optimal PID controller parameters. For future research, it would be interesting to test the new algorithm on the multiobjective optimization problems where more diversity is required.

\section{References}

[1] J.G. Ziegler, N. B. Nichols, Optimum settings for automatic controllers, ASME Trans. (1942) 759-768.

[2] Zwe-Lee Gaing, A particle swarm optimization approach for optimum design of PID controller in AVR system, IEEE Trans. Energy Conv. 19 (2) (2004) 384-391.

[3] Wang Jingi, Xue Yang, Chen Lei, The application of GA-based PID parameter optimization for the control of superheated steam temperature, Int. Conf. on Machine Learning and Cyber. (ICMLC), 2012.

[4] L. S. Coelho, M. W. Pessôa, A tuning strategy for multivariable PI and PID controllers using differential evolution combined with chaotic Zaslavskii map, Expert Systems with Applications 38 (11) ( 2011) 1369413701.

[5] Queen, A.A. ; Auxillia, D.J. ,Simplified discrete binary PSO tuned multivariable PID controller for binary distillation column plant, Int. Conf. on Circuits, Power and Computing Technologies (ICCPCT), March 2013.
[6] J. Lin, J.Huang, G. Chen, Design of DEtuning PID controller for linear servo system, IEEE Fifth Int. Conf. Adv. Comput. Intell. (ICACI), Nanjing ,2012,pp. 437 - 440.

[7] Zhang Jun, Zhang Kanyu, A particle swarm optimization approach for optimal design of PID controller for temperature control in HVAC, Third Int. Conf. on Measuring Technology and Mechatronics Automation (ICMTMA),Shangshai, 2011.

[8] J. Kennedy , R. C. Eberhart, Particle swarm optimization, Proc. IEEE Int. Conf. Neural Netw. ,Perth,1995, pp.1942 - 1948.

[9] R. C. Eberhart, y. shi, PSO: developments, applications and resources, Proc. of the 2001 Cong. on Evol. Comput. ,Seoul, 2001, pp.81-86 .

[10] F. Wang,Xing-shi He,L. Luo, Y. Wang, Hybrid optimization algorithm of PSO and Cuckoo Search,Int conf. on Art. Intell. Manag. Sci.andElectr. Comm., 2011,pp.1172 - 1175.

[11] W. Xu, X. S. Gu, A hybrid particle swarm optimization approach with prior crossover differential evolution, Proc. 1st ACM/SIGEVO Summit Genetic Evol. Comput.,Shanghai, China, 2009, pp. 671-677.

[12] B. B. Li, L.Wang, B. Liu, An effective PSO-based hybrid algorithm for multiobjective permutation flowshop scheduling, IEEE Trans. Syst. Man Cybern. A, Syst. Humans. 38 (4) (2008) 818-831.

[13] C. F. Huang, A hybrid of genetic algorithm and particle swarm optimization for recurrent network design, IEEE Trans. Syst. Man Cybern. B. 34 (2) (2004) 997-1006.

[14] N. Holden , A. A. Freitas, A hybrid particle swarm/ant colony algorithm for the classification of hierarchical biological data, Proc. IEEE Swarm Intell. Symp. ,2005,pp.100-107.

[15] P. S. Shelokar, P. Siarry, V. K. Jayaraman, B. D. Kulkarni, Particle swarm and ant colony algorithms 
hybridized for improving continuous optimization, Appl. Math Comput. 188 (1) (2007) 129-142.

[16] J B. Niu and L. Li, A novel PSO-DEbased hybrid algorithm for global optimization, in: Proc. 4th Int. Conf. Intell. Comput., ,Shanghai, China, 2008, pp.156-163.

[17] H. Zhang, L. Fang, Y. Cen, Comparison among three kinds of hybrid particle swarm optimization algorithms, Cont. and Dec. Conf. (CCDC) ,China,2011, pp.3422 - 3425.

[18] R. Storn, K. Price, Differential evolution - a simple and efficient heuristic for global optimization over continuous spaces, Global Optimization j. 11(4) (1997) 341-359.

[19] S. Das, P. N. Suganthan,Differential Evolution: A survey of the state-of-theart, IEEE Trans. Evol. Comput. 15 (1) (2011) 4-31.

[20] B. Xin, J. Chen, J. Zhang, H. Fang, Z. Peng, Hybridizing differential evolution and particle swarm optimization to design powerful optimizer:a review and taxonomy, IEEE Trans. Syst.ManCybern. $\mathrm{C}$ :application and reviews. 42 (5) (2011)744-767.

[21] S. Das, A. Konar, U. K. Chakraborty, Improving particle swarm optimization with differentially perturbed velocity, in: Proc. Genetic Evol. Comput. Conf. ,Washington, 2005, pp.177-184.

[22] Li Xiao, X. Zuo, Multi-DEPSO: A DE and PSO based hybrid algorithm in dynamic environments, IEEE Cong. Evol. Comput. (CEC), Brisbane , 2012, pp.1-7.

[23] j.Grobler, A.P. Engelbrecht, Hybridizing PSO and DE for improved vector evaluated multi-objective optimization, IEEE Cong. Evol. Comput., Trondheim,2009 , pp. 1255 1262

[24] W. Chen, J. Zhang, Y. Lin, N. Chen, Z. Zhan, H. S. Chung, Y. Li, Y. Shi, Particle swarm optimizer with aging operator for multimodal function optimization, Taylor \& Francis Int. J.
Comput. Intell. Sys. 6 (5) (2013) 862880.

[25]S. Dehuri, A.Ghosh,R.Mall, Particles with age for data clustering, Int. conf. Inform. Tech. (ICIT), 2006, pp.221 224.

[26] R. Kundu, R. Mukherjee, S. Das, Modified Particle Swarm Optimization with Switching Update Strategy, Swarm, Evol., and MemeticComput.,springer,Berlin Heidelberg, 2012, pp 644-652 .

[27] B. jiang, N. Wang, L. Wang, Particle swarm optimization with age- group topology for multimodal functions and data clustering, Comm.. Nonlin. Sci. and Numeric. Sim. 18 (11) (2013) 31343145.

[28] W. Chen, J. Zhang, Y. Lin, N. Chen, Z. Zhan, H. S. Chung, Y. Li, Y. Shi, Particle swarm optimization with an aging leader and challengers, IEEE Trans. Evol. Comput. 17 (2) (2013) 241258.

[29] K.Vaisakh, LR. Srinivas, Adaptive PSODV algorithm for OPF with nonsmooth cost functions and statistical analysis, Simulation Modelling Practice and Theory. 19 (9) (2011) 1824-1846.

[30] K.Vaisakh,L.R.Srinivas,KalaMeah, Genetic evolving ant direction PSODV hybrid algorithm for OPF with nonsmooth cost function, Springer Electr. Eng. 95 (3) (2012) 185-199.

[31] Xiaoli Li, Ji Li, Longhui Shi, Modelling and Simulation of Water Level System, Proceedings of the IEEE Inter. Conf. on Automation and Logistics ,Qingdao, China, 2008,pp. 2857-2859.

[32] B. Kumar,R. Dhiman, Tuning of PID Controller for Liquid Level Tank System using Intelligent Techniques, IJCST. 2 (4) (2011) 257-260.

[33] D. B. Fogel, Evolutionary Computation: Toward a New Philosophy of Machine Intelligence, second ed., IEEE Press, New York, 2000.

[34] $\underline{\text { Z.Hao }}, \underline{\text { G. Guo }}, \underline{\text { H. Huang, A Particle }}$ Swarm Optimization Algorithm with Differential Evolution, Int. Conf. 
Machine Learning and Cyber.,Hong Kong, 2007, pp. $1031-1035$.

[35] N A Selamat, N. A. Wahab, S Sahlan, Particle Swarm Optimization for Multivariable PID Controller Tuning, IEEE 9th Int. Colloquium on Signal Processing and its Applications, Kuala Lumpur, Malaysia, 8 - 10 Mac. 2013.

[36] M. W. I. and S. Baskar, "Evolutionary algorithms based design of multivariable PID controller," Expert Systems with Applications, p. 9, 2009.

[37] A. Visioli, Fuzzy Logic Based Set-Point Weight Tuning of PID Controllers, IEEE Trans. on sys., man, and cybernetics - part a: sys. and humans, 29(6) (1999)587-592.

[38] R. E. Walpole and R. H. Myers, Probability and Statistics for Engineers and Scientist. New York: Macmillan, 1978.

[39]http://www.mathworks.com/help/stats/tt est2.html

[40]K. Ogata, Modern Control Engineering, fifth ed., Prentice Hall, newgersy, 2010.

[41] Y.Jiang, N. R. Chapman and P.Gerstoft , Estimation of Geoacoustic Properties of Marine Sediment Using a Hybrid Differential Evolution Inversion Method, IEEE J. of OCEANIC ENG., (35)(1)( 2010) 59-69.

[42] A. El-Gammal, A.El-Samahy, Adaptive Tuning of a PID Speed Controller for DC Motor Drives Using Multi-Objective Particle Swarm Optimization, IEEE UKSim 2009: 11th Int.Conf. on Computer Modelling and Simulation, Cambridge, 2009,pp.398-404.

[43] M. A. Elhosseini, A.Y. Haikal, Modified hybrid algorithm for process optimization, App. Math. andComput., 232 (2014) 1209-1224.

[44]http://www.mathworks.com/help/gads/in dex.html. 